\title{
Preparation of silk fibroin microspheres by emulsification- diffusion method for controlled release drug delivery applications
}

\author{
Thanonchat Imsombut, Mangkorn Srisa-ard, Prasong Srihanam, Yodthong Baimark \\ *Department of Chemistry and Center of Excellence for Innovation in Chemistry, \\ Faculty of Science, Mahasarakham University, Mahasarakham 44150, Thailand; e- \\ mail: yodthong.b@msu.ac.th.
}

(Received: 27 November, 2010; published: 01 September, 2011)

\begin{abstract}
Silk fibroin (SF) microspheres containing a model drug were prepared by a simple water-in-oil emulsification-diffusion method. Aqueous SF solution and ethyl acetate were used as water and oil phases, respectively. Methylene Blue was used as a water-soluble model drug. Effect of drug content on microsphere characteristics, drug loading efficiency and drug release behavior was determined. Scanning electron microscopy revealed that drug-loaded SF microspheres were spherical in shape with a smooth surface. The SF microspheres showed predominant random coil SF conformation. All drug loading efficiencies were approximately $50 \%$. An increase in drug/SF ratio could lead to an increase in $\%$ drug release and surface erosion after the drug release test. Drug release from SF microspheres is due to SF matrix swelling and surface erosion processes.
\end{abstract}

\section{Introduction}

Silk fibroin (SF) from Bombyx mori fibres has been extensively investigated as a promising biomaterial because of its biocompatibility and biodegradability [1]. For medical and pharmaceutical applications, regenerated SF devices have been prepared in several forms of fibres [2-4], films [5, 6], nanoparticles [7] and microparticles [8].

Biodegradable microspheres are often proposed for use as controlled release drug delivery systems due to their well-defined model for degradation and drug release. Spray drying, ball-milling, lipid templating and emulsification methods have been reported for preparing SF microspheres [9-14]. However, a suitable method for production of the SF microspheres for drug delivery remains to be identified. From our previous works $[15,16]$, the water-in-oil (W/O) emulsification-diffusion method has been used to prepare the SF microparticles. For this purpose, the W/O emulsion system is composed of an aqueous SF solution (dispersed water phase) and a continuous oil phase, organic solvent. When ethyl acetate was used as an oil phase, SF microparticles with irregular shapes were obtained. The SF microparticles were formed after diffusion out of water from the W/O emulsion droplets to continuous phase, ethyl acetate. Span80 dissolved in the ethyl acetate phase can induce SF microspheres which from irregular to spherical shapes [17].

Herein, we have developed a W/O emulsification-diffusion method for preparing drug-loaded SF microspheres. Aqueous SF solution and ethyl acetate were used as water and oil phases, respectively. The influence of Methylene Blue (model drug) 
content on SF microsphere characteristics and drug release behaviors was determined.

\section{Results and discussion}

Morphology and sizes of MB-loaded SF microspheres

SEM micrographs of the shapes and surfaces of drug-loaded SF microspheres are illustrated in Figures 1 and 2, respectively.

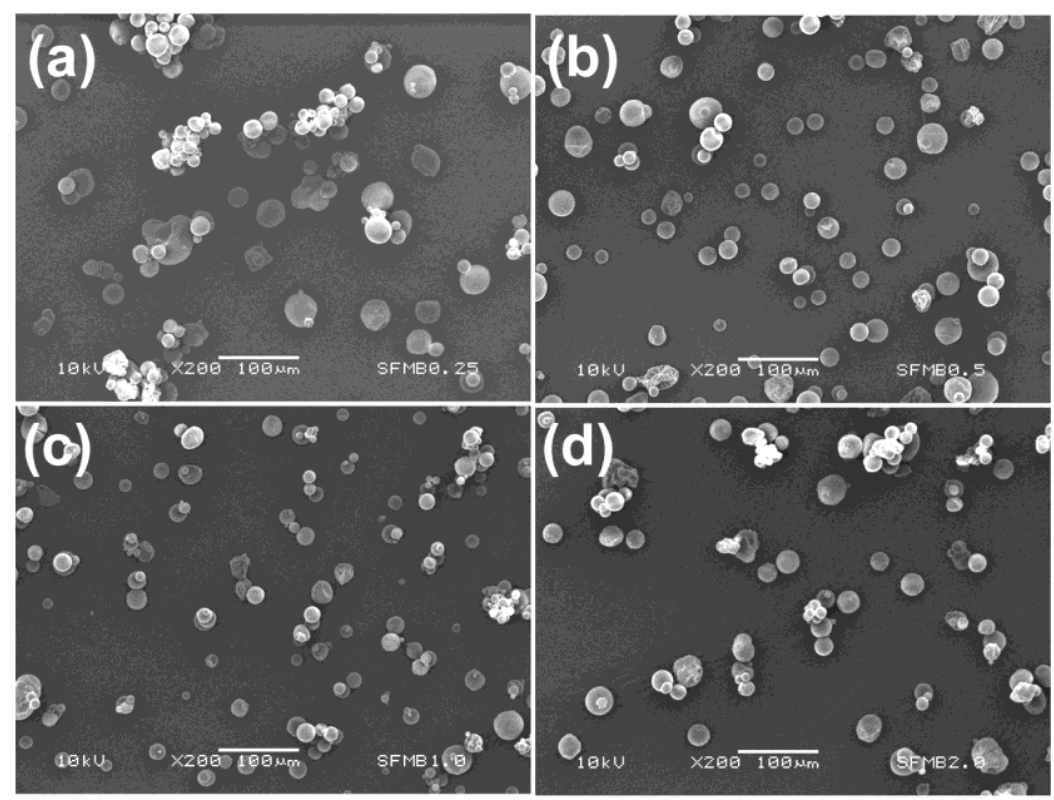

Fig. 1. SEM micrographs of MB-loaded SF microspheres prepared using MB/SF ratios of (a) $0.25 \%$, (b) $0.5 \%$, (c) $1 \%$ and (d) $2 \% \mathrm{w} / \mathrm{w}$. All bars $=100 \mu \mathrm{m}$.

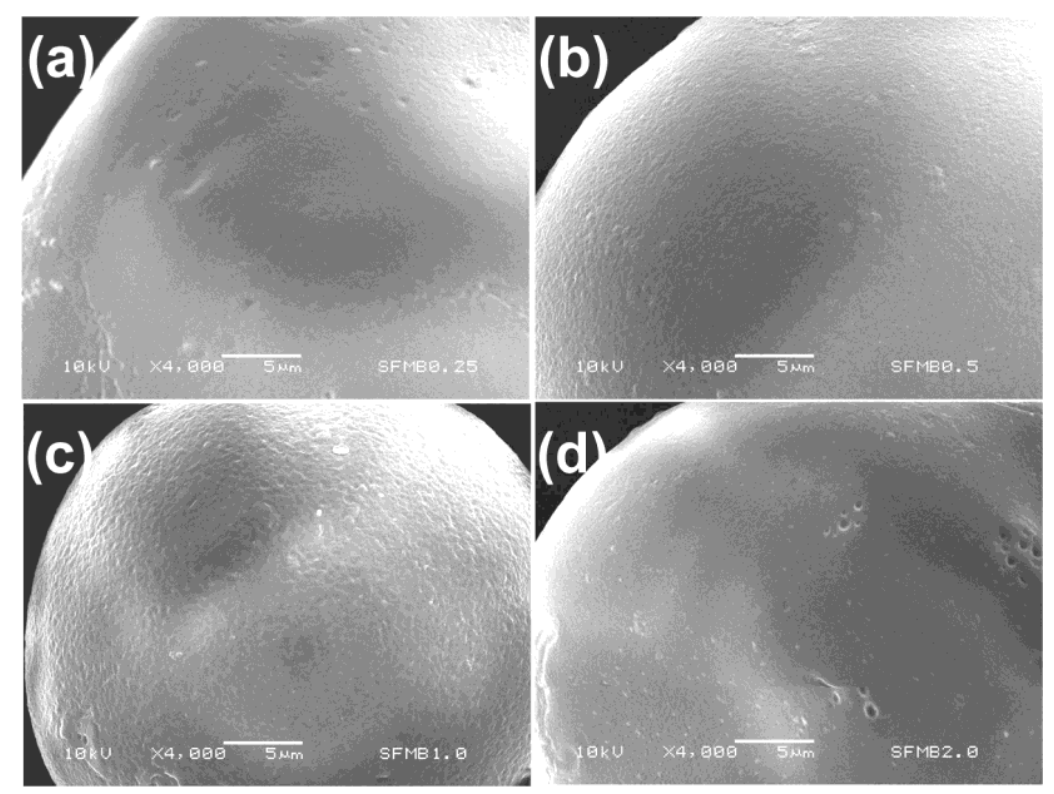

Fig. 2. SEM micrographs of surfaces of MB-loaded SF microspheres prepared using $\mathrm{MB} / \mathrm{SF}$ ratios of (a) $0.25 \%$, (b) $0.5 \%$, (c) $1 \%$ and (d) $2 \% \mathrm{w} / \mathrm{w}$. All bars $=5 \mu \mathrm{m}$. 


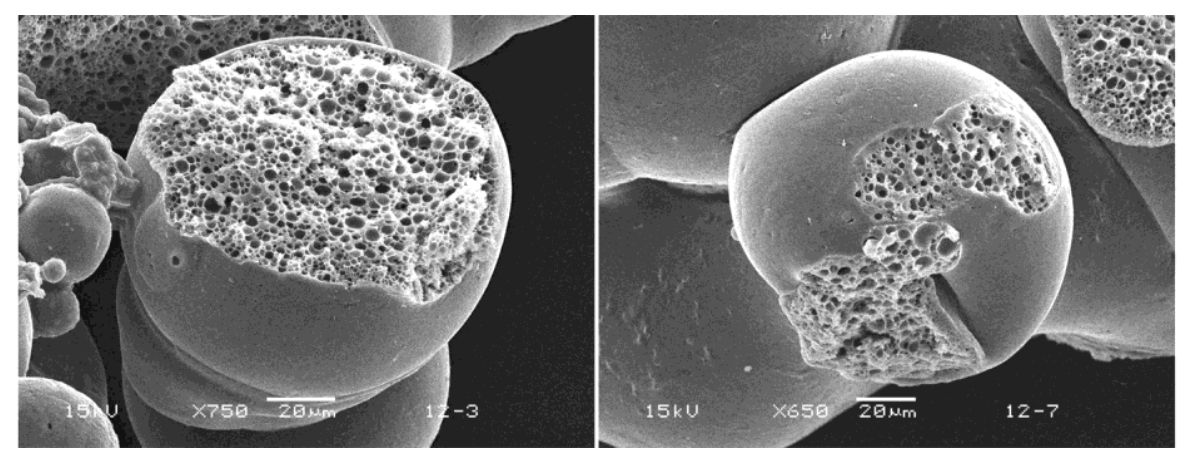

Fig. 3. SEM micrographs of matrices of MB-free (left) and $2 \% \mathrm{w} / \mathrm{w}$ MB-loaded (right) SF microspheres. All bars $=20 \mu \mathrm{m}$.

It can be seen that drug-loaded SF microspheres are spherical in shape and had a smooth surface. This indicates that the Methylene Blue (MB) entrapment did not affect shape and surface roughness of the SF microspheres. Internal morphology of microspheres can be observed through their broken surfaces, examples of which are shown in Figure 3. Internal porous structures covered with a continuous smooth surface were found for both drug-free and drug-loaded SF microspheres. This may be explained due to phase separation take place in emulsion droplets before microsphere solidification. The porous structure could form when a small amount of non-solvent (ethyl acetate) diffused into each SF emulsion droplet and worsened the solubility of the SF. Then SF solidified and precipitated rapidly predominant in a random coil conformation. The internal porous structures of SF microspheres did not change when the MB was entrapped.

Table 1 reports drug loading content (DLC), drug loading efficiency (DLE) and average particle sizes of the MB-loaded SF microspheres. The DLC $\mathrm{Catual}_{\text {values }}$ increased according to the $\mathrm{DLC}_{\text {feed }}$ values. Thus the drug-loaded SF microspheres with different $\mathrm{MB}$ contents can be prepared by using different weights of the feed MB. The DLE values indicated the efficiency of the technique to entrap the drug within the microspheres was in the range of $45-53 \%$. From Table 1, the DLE did not depend upon the $\mathrm{DLC}_{\text {feed. }}$ Some MB can diffuse out from the emulsion droplets to the ethyl acetate continuous phase during the diffusion step. MB-loaded SF microsphere sizes were in the range of $25-31 \mu \mathrm{m}$ which did not change in significantly when the MB was entrapped and MB/SF ratio was increased up to $2 \% \mathrm{w} / \mathrm{w}$.

Tab. 1. Drug loading efficiency of MB-loaded SF microspheres.

\begin{tabular}{ccccc}
\hline $\begin{array}{c}\text { MB/SF ratio } \\
(\% \text { w/w })\end{array}$ & $\begin{array}{c}\mathrm{DLC}_{\text {feed }}{ }^{\mathrm{a}} \\
(\%)\end{array}$ & $\begin{array}{c}\mathrm{DLC}_{\text {actual }}{ }^{\mathrm{b}} \\
(\%)\end{array}$ & $\begin{array}{c}\mathrm{DLE}^{\mathrm{c}} \\
(\%)\end{array}$ & $\begin{array}{c}\text { Average size }^{\mathrm{d}} \\
(\mu \mathrm{m}) \pm \mathrm{SD}\end{array}$ \\
\hline 0 & - & - & - & $24 \pm 9$ \\
0.25 & 0.25 & 0.13 & 52 & $31 \pm 9$ \\
0.5 & 0.50 & 0.25 & 50 & $25 \pm 11$ \\
1 & 0.99 & 0.45 & 45 & $30 \pm 12$ \\
2 & 1.96 & 1.03 & 53 & $28 \pm 15$ \\
\hline
\end{tabular}

\footnotetext{
${ }^{a}$ Feed drug loading content calculated from equation (3.2).

${ }^{\mathrm{b}}$ Actual drug loading content calculated from equation (3.1).

${ }^{\mathrm{C}}$ Drug loading efficiency calculated from equation (3.3).

${ }^{d}$ Measured from several SEM images.
} 
Figure 4 shows FTIR spectra of drug-loaded SF microspheres [Figures 4(b) - 4(e)] compared to drug-free SF microspheres [Figure 4(a)]. The MB powder exhibits symmetric stretch of C-N at $1398 \mathrm{~cm}^{-1}$ and asymmetric deformation of $-\mathrm{CH}_{3}$ at 1344 $\mathrm{cm}^{-1}$ in its FTIR spectrum. The FTIR spectra of drug-loaded SF microspheres showed MB characteristics at $1398-1399 \mathrm{~cm}^{-1}$ (C-N stretching) and $1344-1345 \mathrm{~cm}^{-1}\left(-\mathrm{CH}_{3}\right.$ deformation). The wave numbers of amide I, II and III bands in FTIR spectra of the drug-loaded SF microspheres indicated that all drug-loaded SF microspheres showed predominantly random coil SF form. The MB loading did not induce SF conformational changes from random coil to $\beta$-sheet form.

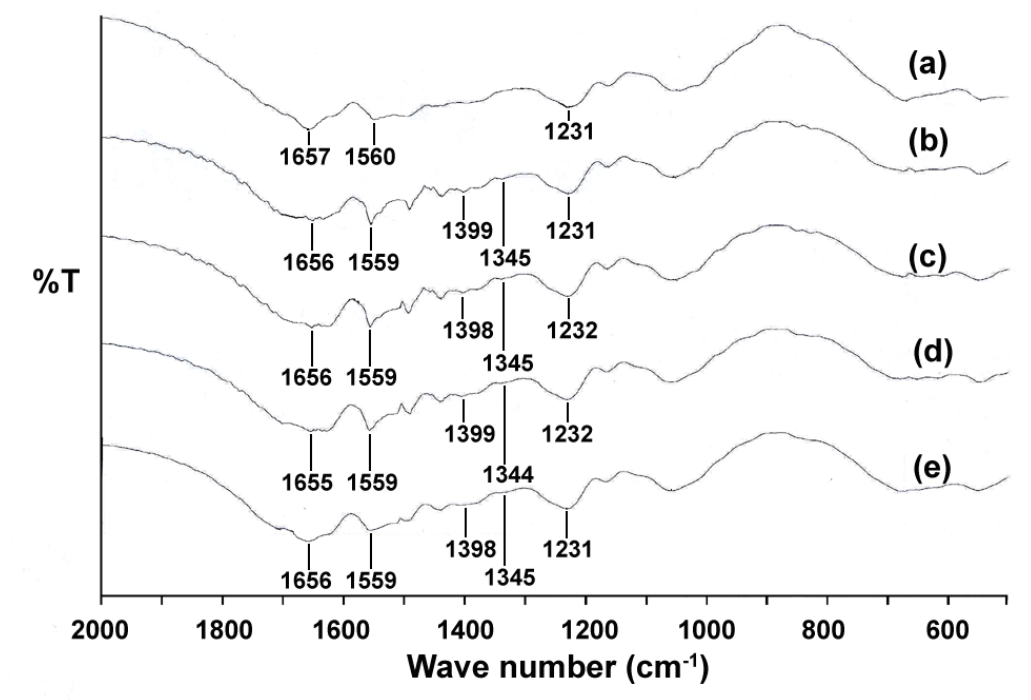

Fig. 4. FTIR spectra of (a) MB-free SF microspheres and MB-loaded SF microspheres with MB/SF ratios of (b) $0.25 \%$, (c) $0.5 \%$, (d) $1 \%$ and (e) $2 \% \mathrm{w} / \mathrm{w}$.

The in vitro release profiles of MB from the SF microspheres are shown in Figure 5. MB was selected as a model of water-soluble drug to investigate the effect of drug/SF ratio on the drug release behavior from the SF microspheres. All MB/SF ratios of the SF microspheres clearly showed controlled release patterns in the first $12 \mathrm{~h}$ of releasing time followed by further slow release. It can be seen from Figure 5 that the release profiles of $1 \%$ and $2 \% \mathrm{w} / \mathrm{w} \mathrm{MB} / \mathrm{SF}$ microspheres are similar. The initial burst release rate increased significantly when the MB/SF ratio increased from $0.25 \%$ to $0.5 \%$ and $1 \% \mathrm{w} / \mathrm{w}$.

The drug release mechanism from a biodegradable polymer matrix consisted of two main processes, which are outwards drug diffusion due to swelling of the polymer matrix (swelling-controlled system) and drug release due to degradation and erosion of polymer matrix (erosion-controlled system) [18]. The drug release results suggest that the drug release in the first $12 \mathrm{~h}$ is due to the drug concentration gradient by matrix swelling. Swelling behaviour of the SF matrix directly related to the MB/SF ratio. This may be explained by the high amount of feed MB $(2 \% \mathrm{w} / \mathrm{w})$ which could affect the properties of the SF matrix network, thus affecting the diffusion barrier. This indicates that the drug release behaviors of the initial burst release were controlled by adjusting the drug/SF ratio. In addition, the slow release step of MB may occur by surface erosion of the SF matrix. Figure 6 shows degradation and erosion of 
microsphere surfaces after the drug release test, which were rougher than those of before the drug release test.

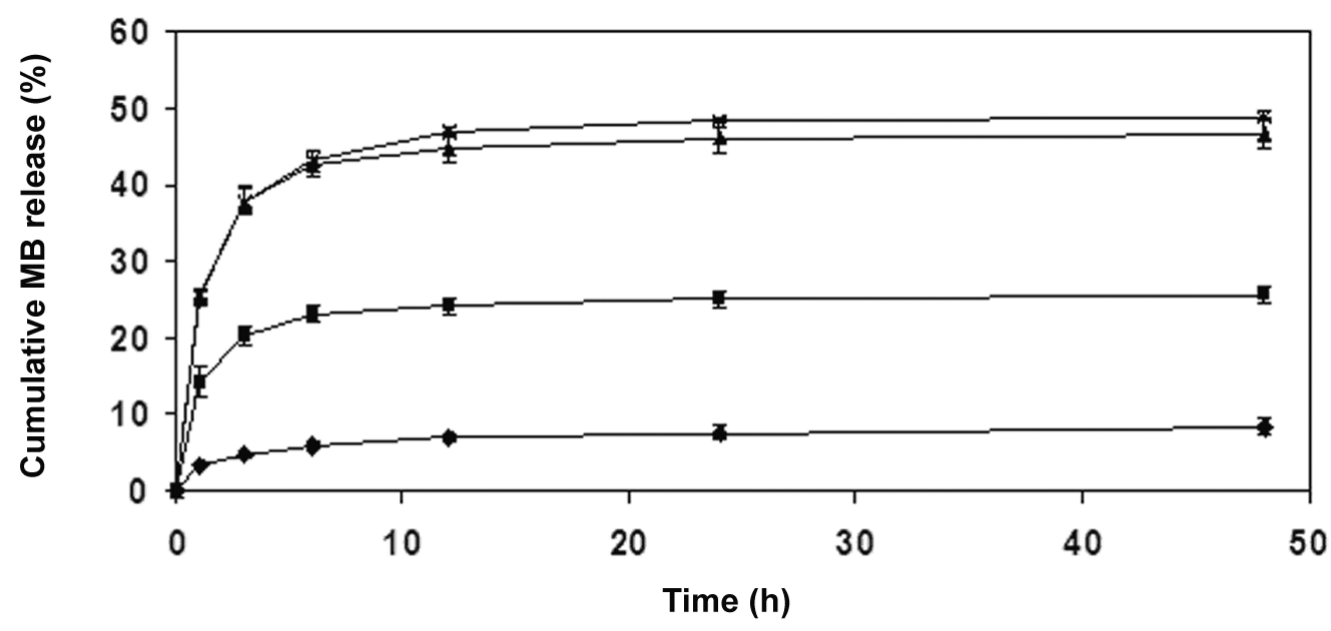

Fig. 5. In vitro $\mathrm{MB}$ release from MB-loaded SF microspheres prepared using MB/SF ratios of $(\bullet) 0.25 \%,(\boldsymbol{a}) 0.5 \%,(\Delta) 1 \%$ and $(\times) 2 \% \mathrm{w} / \mathrm{w}$.

The general expression $Q_{t} / Q_{0}=k t^{n}$ was derived for drug release from polymer matrices by coupling a Fickian (diffusion-controlled) and non-Fickian mechanism [19], where $Q_{t} / Q_{0}$ is the fractional solute release, $t$ is the release time, $k$ is a constant, and $n$ is the diffusional exponent characteristic of the release mechanism. For diffusioncontrolled release and zero-order release are defined by $n=0.5$ and $n=1.0$, respectively.
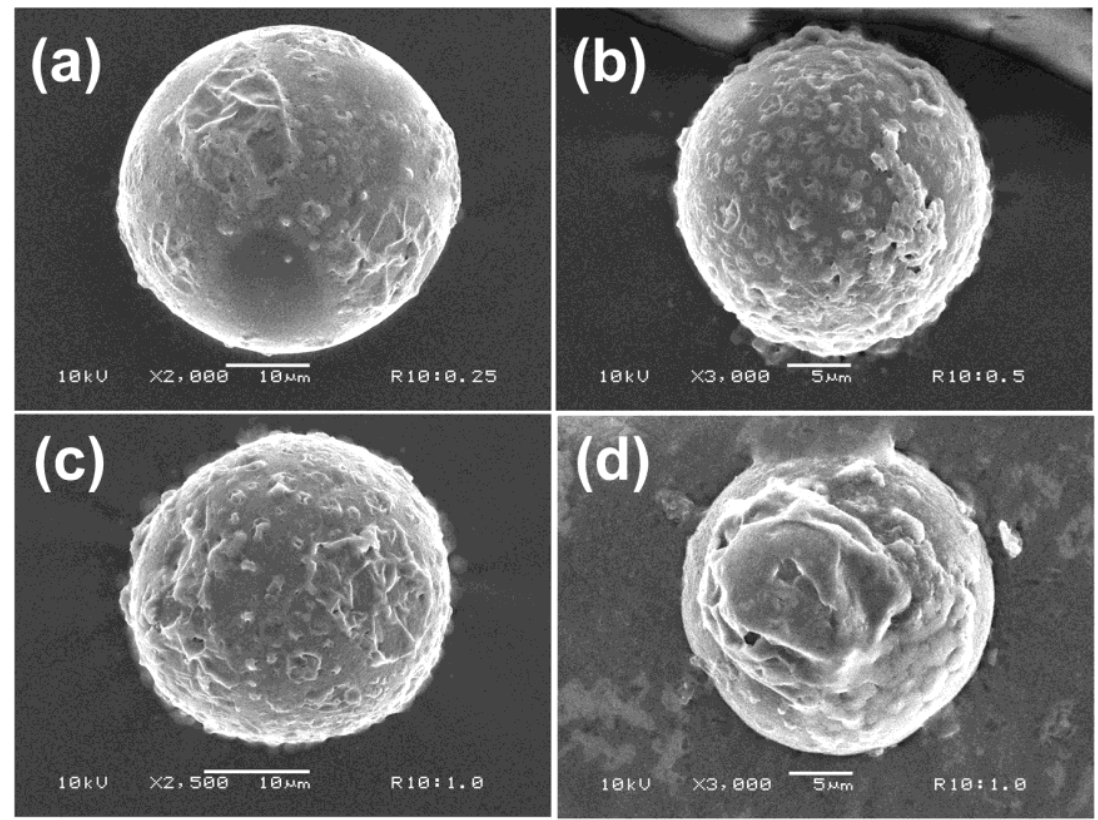

Fig. 6. SEM micrographs after $48 \mathrm{~h}$ drug release test of MB-loaded SF microspheres prepared using MB/SF ratios of (a) $0.25 \%$, (b) $0.5 \%$, (c) $1 \%$ and (d) $2 \% \mathrm{w} / \mathrm{w}$. Bars = $10 \mu \mathrm{m}$ for (a) and (c), and bars $=5 \mu \mathrm{m}$ for (b) and (d). 
The application of the equation to porous matrices where the drug release occurs by diffusion partially through a swollen matrix and partially through water-filled holes would probably lead to $n<0.5$ [20]. In this work, the $n$ values of SF microspheres entrapped with $0.25 \%, 0.5 \%, 1 \%$ and $2 \%(\mathrm{w} / \mathrm{w}) \mathrm{MB}$ were $0.26,0.14,0.15$ and 0.16 , respectively. This indicated that the diffusion controlled release kinetic through a swollen SF microsphere matrix and water-filled holes may play an important role in the release behavior of these SF microspheres.

\section{Experimental part}

\section{Materials}

Cocoons of Bombyx mori silk were de-gummed to extract the glue-like sericin proteins and wax before dissolving [21]. De-gumming, silk cocoons $(5 \mathrm{~g})$ were boiled three times in $500 \mathrm{~mL}$ of $0.5 \% \mathrm{w} / \mathrm{w} \mathrm{Na} \mathrm{Na}_{2} \mathrm{CO}_{3}$ aqueous solution at $85{ }^{\circ} \mathrm{C}$ for $30 \mathrm{~min}$ each time. The silk fibroin (SF) fibers of de-gummed cocoons were then rinsed thoroughly with water before being dried at $30{ }^{\circ} \mathrm{C}$ overnight. The dried SF fibers $(5 \mathrm{~g})$ were dissolved in $100 \mathrm{~mL}$ of $\mathrm{CaCl}_{2}$-ethanol-water (1-2-8 mole ratio) mixture at $85^{\circ} \mathrm{C}$ until total dissolution. This SF solution was dialyzed against $2 \mathrm{~L}$ of distilled water for 3 days using a dialysis tube (molecular weight cut off: 7,000 Da, $22 \mathrm{~mm}$ in dry diameter, Lot \# KC132124, Thermo Scientific Co. Ltd.). The distilled water was changed every day. The obtained SF concentration was determined by weighting the remaining solid after drying at $60{ }^{\circ} \mathrm{C}$ overnight. The final SF concentration was adjusted to $1.0 \%(\mathrm{w} / \mathrm{v})$ with distilled water. Ethyl acetate (AR, Lab Scan) was used as a continuous oil phase. Span80 (Merck) and Methylene Blue (MB, Carlo Erba) were used as oil-soluble emulsifier and water-soluble model drug, respectively.

\section{Preparation of drug-loaded SF microspheres}

SF microspheres containing MB were prepared by the water-in-oil emulsion solvent diffusion method. An appropriate amount of $\mathrm{MB}$ was directly dissolved in the SF solution before microsphere formation. One $\mathrm{mL}$ of SF and MB solution was addeddrop wise with a speed of $1 \mathrm{~mL} / \mathrm{min}$ to $200 \mathrm{~mL}$ of $2 \%(\mathrm{w} / \mathrm{v})$ Span80 solution in ethyl acetate under magnetic stirring at $900 \mathrm{rpm}$. A $1 \mathrm{~mL}$ pipette was used for this purpose. After emulsification-diffusion process which lasted for $1 \mathrm{~h}$, the resultant drug-loaded SF microspheres suspended in ethyl acetate were collected by centrifugation before rinsing with fresh ethyl acetate twice and drying in a vacuum oven for overnight.

\section{Characterization of drug-loaded SF microspheres}

Morphology of the SF microspheres was studied by scanning electron microscopy (SEM) using a JEOL JSM-6460LV SEM. The microspheres were sputter coated with gold for enhanced conductivity before scan. Average particle sizes and standard deviation (SD) were measured from particle diameter and determined from several SEM images of each sample by manually counting a minimum of 150 particles using smile view software (version 1.02, JEOL Ltd.).

Chemical structure and SF conformation of the SF microspheres were investigated by Fourier transform infrared (FTIR) spectroscopy using a Perkin-Elmer Spectrum GX FTIR spectrometer with air as the reference. A resolution of $4 \mathrm{~cm}^{-1}$ and 32 scans were chosen. FTIR spectra were obtained from a KBr disk method. 
Actual drug loading content (DLC actual) of the drug-loaded SF microspheres was measured by dissolving it in distilled water with shaking for a week. MB content was determined by UV-Vis spectrophotometer at $\lambda_{\max }=665 \mathrm{~nm}$. \%DLC was calculated from Equation (1). The \%DLC value is the average of three different determinations. Feed drug loading content ( $\mathrm{DLC}_{\text {feed }}$ ) was calculated from Equation (2). Drug loading efficiency, ratio of $\mathrm{DLC}_{\text {actual }}$ and $\mathrm{DLC}_{\text {feed }}$ was calculated from Equation (3).

$\% \mathrm{DLC}_{\text {actual }}=$

[Weight of entrapped drug/Weight of drug-loaded SF microspheres] $\times 100$

$\% \mathrm{DLC}_{\text {feed }}=[$ Weight of feed drug/Weights of feed drug and SF $] \times 100$

$\% \mathrm{DLE}=\left[\% \mathrm{DLC}_{\text {actual }} / \% \mathrm{DLC} \mathrm{C}_{\text {feed }}\right] \times 100$

In vitro drug release test

In vitro drug release test was performed in $1 \mathrm{~mL}$ of $0.1 \mathrm{M}$ phosphate buffer solution $\mathrm{pH} 7.4$ at $37^{\circ} \mathrm{C}$ under shaking at $100 \mathrm{rpm}$. The $15 \mathrm{mg}$ of each microsphere sample was used for drug release testing. At each interval time, drug solution was collected after centrifugation at 10,000 rpm for $5 \mathrm{~min}$. One $\mathrm{mL}$ of fresh phosphate buffer solution was added. MB concentration was measured by UV-Vis spectrophotometry at $\lambda_{\max }=665 \mathrm{~nm}$. Each sample was run in triplicate. The \%drug release was plotted with time.

\section{Acknowledgements}

This work was supported by Mahasarakham University (fiscal year 2011), The National Metal and Materials Technology Center (MTEC), National Science and Technology Development Agency (NSTDA), Ministry of Science and Technology, Thailand (MT-B-52-BMD-68-180-G) and The Center of Excellence for Innovation in Chemistry (PERCH-CIC), Commission on Higher Education, Ministry of Education, Thailand.

\section{References}

[1] Altman, G. H.; Diaz, F.; Jakuba, C.; Calabro, T.; Horan, R. L.; Chen, J.; Lu, H.; Richmond, J.; Kaplan, D. L. Biomaterials 2003, 24, 401.

[2] Wang, M.; Jin, H. J.; Kaplan, D. L.; Rutledge, G. C. Macromolecules 2004, 37, 6856.

[3] Li, C.; Vepari, C.; Jin, H. J.; Kim, H. J.; Kaplan, D. L. Biomaterials 2006, 27, 3115.

[4] Weiwei, B.; Youzhu, Z.; Guibo, Y.; Jialin, W. e-Polymers 2008, no. 098.

[5] Liu, Y.; Liu, H.; Qian, J.; Deng, J.; Yu, T. J. Macromol. Sci. A 1996, 33, 209.

[6] Jin, H. J.; Park, J.; Valluzzi, R.; Cebe, P.; Kaplan, D. L. Biomacromolecules 2004, 5,711 .

[7] Zhang, Y. Q.; Shen, W. D.; Xiang, R. L.; Zhuge, L. J.; Gao, W. J.; Wang, W. B. J. Nanopart. Res. 2007, 9, 885.

[8] Wang, X.; Wenk, E.; Matsumoto, A.; Meinel, L.; Li, C.; Kaplan, D. L. J. Controlled Release 2007, 117, 360.

[9] Yeo, J. H.; Lee, K. G.; Lee, Y. W.; Kim, S. Y. Eur. Polym. J. 2003, 39, 1195.

[10] Hino, T.; Tanimoto, M.; Shimabayashi, S. J. Colloid Interf. Sci. 2003, 266, 68.

[11] Wang, X.; Wenk, E.; Matsumoto, A.; Meinel, L.; Li, C.; Kaplan, D. L. J. Controlled

Release 2007, 117, 360.

[12] Rajkhowa, R.; Wang, L.; Wang, X. Powder Technol. 2008, 185, 87. 
[13] Rajkhowa, R.; Wang, L.; Kanwar, J.; Wang, X. Powder Technol. 2009, 191, 155.

[14] Srisuwan, Y.; Srihanam, P.; Baimark, Y. J. Macromol. Sci. A 2009, 46, 521.

[15] Baimark, Y.; Srihanam, P. J. Appl. Sci. 2009, 9, 3876.

[16] Baimark, Y.; Srihanam, P.; Srisuwan, Y.; Phinyocheep, P. J. Appl. Polym. Sci. 2010, 118, 1127.

[17] Imsombut, T.; Srisuwan, Y.; Srihanam, P.; Baimark, Y. Powder Technol. 2010, 203, 603.

[18] Arifin, D. Y.; Lee, L. Y.; Wang, C-. H. Adv. Drug Deliver. Rev. 2006, 58, 1274.

[19] Zulegar, S.; Lippold, B. C. Int. J. Pharm. 2001, 217, 139.

[20] Medlicott, N. J.; Tucker, I. G.; Rathbone, M. J.; Holborow, D. W.; Jones, D. S. Int. J. Pharm. 1996, 143, 25.

[21] Nogueira, G. M.; Rodas, A. C. D.; Leite, C. A. P.; Giles, C.; Higa, O. Z.; Polakiewicz, B.; Beppu, M. M. Bioresource Technol. 2010, 101, 8446. 